\title{
Dimer formation in nicotinamide and picolinamide in the gas and condensed phases probed by infrared spectroscopy $\dagger$
}

\author{
Ana Borba, ${ }^{a}$ Merwe Albrecht, ${ }^{b}$ Andrea Gómez-Zavaglia, ${ }^{a c}$ Leszek Lapinski, ${ }^{d}$ \\ Maciej J. Nowak, ${ }^{d}$ Martin A. Suhm ${ }^{b}$ and Rui Fausto* ${ }^{a}$
}

Received 12th June 2008, Accepted 22nd August 2008

First published as an Advance Article on the web 16th October 2008

DOI: $10.1039 / \mathbf{b 8 1 0 0 0 2 k}$

\begin{abstract}
Aggregation of nicotinamide (3-pyridine-carboxamide; NA) and picolinamide (2-pyridinecarboxamide; PA) has been investigated by matrix-isolation, supersonic jet and neat solid state infrared spectroscopy, complemented by DFT(B3LYP)/6-311 + $+\mathrm{G}(\mathrm{d}, \mathrm{p})$ calculations. For both compounds, the most stable dimeric structure was shown to be the centrosymmetric dimer where two monomers in their most stable forms establish two $\mathrm{NH} \cdots \mathrm{O}=\mathrm{C}$ hydrogen bonds. The most stable structures of monomers of NA and PA were characterized in detail experimentally by matrix-isolation spectroscopy and theoretically (at both the DFT(B3LYP)/6-311++G(d,p) and MP2/6-311 + + G(d,p) levels). For nicotinamide, two conformers were found in the matrices, with ca. $80 \%$ of the total population adopting the $E$ form. The monomers and dimers of PA and NA were also investigated by infrared spectroscopy of the studied compounds seeded in supersonic jet expansions. These studies revealed that the constraints on the vibrational dynamics in the PA dimer are different from those in the NA dimer. In the PA dimer, the vibrational energy flow out of the $\mathrm{N}-\mathrm{H}$ stretching mode was shown to be accelerated substantially by the presence of a secondary intramolecular hydrogen bond. In the glassy state of both compounds, the centrosymmetric dimer seems to be the prevalent structure. In the neat crystalline state $(\mathrm{KBr}$ pellet), picolinamide keeps this type of dimeric structure as the constituting unit, whereas nicotinamide molecules assume a different arrangement where one of the $\mathrm{NH} \cdots \mathrm{O}=\mathrm{C}$ bonds is replaced by an $\mathrm{NH} \cdots \mathrm{N}$ (ring) bond. The different crystallograpic structures which were formed by the compounds are reflected in the vibrational spectra of the solids. These observations are correlated with the molecular properties of NA and PA, in particular with the greater conformational mobility of NA compared with PA. This is ascribable to the absence in the NA molecule of the intramolecular $\mathrm{NH} \cdots \mathrm{N}_{(\text {ring) }}$ interaction, which exists in PA.
\end{abstract}

\section{Introduction}

Nicotinamide (3-pyridine-carboxamide; NA) and picolinamide (2-pyridine-carboxamide; PA) are pyridine derivatives bearing a carboxamide group at $\beta$ and $\alpha$ positions, respectively (Scheme 1). Both compounds show important biological activity. ${ }^{1-10}$ Nicotinamide is a form of Vitamin $B_{3}$ essential for growth and conversion of food into energy. Due to its role in the improvement of beta cell function, it has been used in the diabetes treatment and prevention. ${ }^{1,2}$ Nicotinamide also exhibits antioxidant, anti-inflammatory and anticarcinogenic activities, as well as putative activity against osteoarthritis and granuloma annulare. ${ }^{3,4}$ In addition, it finds application, for example, in dietary food, nutritional ingredients and cosmetics. ${ }^{5,6}$ Via its major metabolite, NAD (nicotinamide adenine dinucleotide), NA is also involved in a wide range

\footnotetext{
${ }^{a}$ Department of Chemistry, University of Coimbra, Portugal.

E-mail:rfausto@ci.uc.pt

${ }^{b}$ Institute of Physical Chemistry, University of Göttingen, Germany

${ }^{c}$ Facultad de Farmacia y Bioquimica, Universidad de Buenos Aires, Argentina

${ }^{d}$ Institute of Physics, Polish Academy of Science, Warsaw, Poland

$\dagger$ Electronic supplementary information (ESI) available: Additiona

experimental and computational details. See DOI: 10.1039/b810002k
}

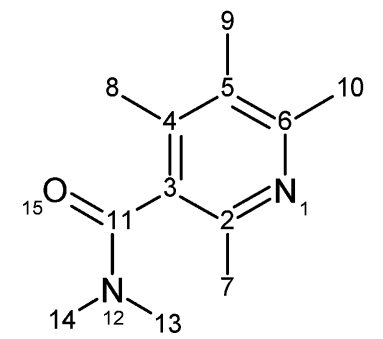<smiles>[3H][Y1](=O)C1=S([3H])C([B])=[SH](C)=N1</smiles>

Scheme 1 Most stable forms $(E)$ of nicotinamide (left) and picolinamide (right) with an adopted atom numbering scheme (the chemical symbols for carbon and hydrogen atoms are omitted for simplicity; carbon atoms correspond to the numbers 2, 3, 4, 5, 6 and 11 ; hydrogens to $7,8,9,10,13$ and 14).

of biological processes: production of energy, synthesis of fatty acids, cholesterol and steroids, signal transduction and maintenance of the integrity of the genome. ${ }^{7-9} \mathrm{PA}$ was found to be a strong inhibitor of poly(ADP-ribose) synthetase. ${ }^{10}$

The monomeric structures of these two compounds, as well as their crystallographic arrangements, have already been a subject of several investigations. NA was studied in the gas phase by electron diffraction ${ }^{11}$ and microwave spectroscopy. ${ }^{12}$ According to these studies, ${ }^{11,12}$ NA exists in the gas phase as a 


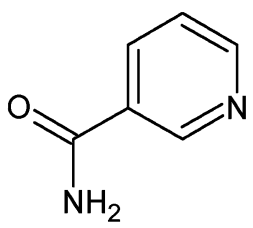

E

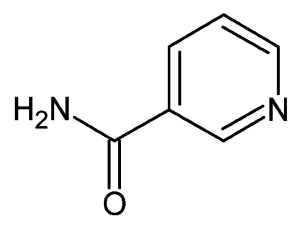

Z
Scheme 2 Schematic representation of the $E$ and $Z$ conformers of nicotinamide.

mixture of two conformers, $E$ and $Z$ (Scheme 2), in which the planar carboxamide and pyridyl moieties are skewed in relation to each other. The $\mathrm{C}_{2}-\mathrm{C}_{3}-\mathrm{C}_{11}-\mathrm{O}_{15}$ dihedral angle (see Scheme 1 for atom numbering) in the $E$ and $Z$ forms was found by microwave spectroscopy ${ }^{12}$ to be 166 and $22^{\circ}$, respectively (146 and $37^{\circ}$, by electron diffraction ${ }^{11}$ ). In a microwave spectroscopy study, ${ }^{12}$ the $E$ conformer was proposed to constitute $c a .95 \%$ of the total population in the supersonic jet expanding from a nozzle heated to $c a .170{ }^{\circ} \mathrm{C}$. Depending on the conformational cooling taking place in the jet, this corresponds to an energy difference between the $Z$ and $E$ forms in the $6.6-12.6 \mathrm{~kJ} \mathrm{~mol}^{-1}$ range. ${ }^{12}$ Infrared spectroscopy studies $^{13-16}$ and dipole moment measurements ${ }^{17,18}$ on NA and some of its deuterated isotopologues also consistently found $\mathrm{NA}$ as existing essentially in the skewed $E$ conformation under different experimental conditions.

In contrast to NA, no previous gas phase studies have been reported for PA. On the other hand, this compound has recently been studied by some of us by matrix isolation and low temperature solid-state infrared spectroscopy and quantum chemical calculations. ${ }^{19}$ In cryogenic rare gas matrices, the monomer of the compound was found to exist in a single planar conformation, where the two nitrogen atoms are in syn periplanar geometry ( $E$; see Scheme 1). This conformer is stabilized by the presence of two intramolecular hydrogen-bond-type interactions ( $\mathrm{NH} \cdots \mathrm{N}$ and weak $\mathrm{C}=\mathrm{O} \cdots \mathrm{H}$ ) and was predicted theoretically to be more stable than the second conformer $(Z)$ by more than $35 \mathrm{~kJ} \mathrm{~mol}^{-1}$. These results are in agreement with previous ${ }^{1} \mathrm{H}$ NMR studies $^{20,21}$ that had suggested, on the basis of the observed chemical shifts of the amide protons, that the molecule should be planar and bear an intramolecular hydrogen bond between the $-\mathrm{NH}_{2}$ amide group and the pyridyl nitrogen atom.

The fact that the preferred crystalline arrangements in $\mathrm{NA}^{22,23}$ and $\mathrm{PA}^{24}$ (whose molecular structures are closely related) involve different basic units, with the centrosymmetric dimer existing in the two known crystalline modifications of $\mathrm{PA}^{24}$ and being absent in the crystal of $\mathrm{NA},{ }^{22,23}$ caught our attention and stimulated us to investigate the structures and relative energies of the relevant dimeric structures in these two molecules. The case of NA appeared particularly interesting, since it might be an exception to the general tendency of amides to form centrosymmetric dimers. ${ }^{25-27}$ Matrix-isolation, supersonic jet and neat solid state infrared spectroscopy, complemented by DFT(B3LYP)/6-311 + + G(d,p) calculations, were then used in a combined way, in order to establish the nature of the most stable structure of the dimers of the two studied compounds and to characterize the most important factors which determine their stability. As shown below, the different behaviour of the two compounds upon crystallization is reflected in the vibrational spectra of their solid samples and can be correlated with the greater conformational mobility of NA compared with PA, due to the absence in the first compound of the intramolecular $\mathrm{NH} \cdots \mathrm{N}_{(\mathrm{pyr})}$ interaction, which exists in PA.

\section{Experimental and computational methods}

\section{Infrared spectroscopy}

Commercial samples of nicotinamide $(>97 \%)$ and picolinamide $(>98 \%)$ were used in the present studies. As mentioned in the introduction, PA has been studied recently by matrix isolation and neat solid state infrared spectroscopy by some of us, ${ }^{19}$ so that in the present investigation only the jet spectroscopy studies, described below, were performed on this compound. To obtain the NA matrices a solid sample of this compound was electrically heated in a miniature glass oven placed in the vacuum chamber of the cryostat (either a continuous-flow helium cryostat or an APD Cryogenics close-cycle helium refrigeration system with a DE-202A expander). The vapour of the compound was deposited, together with a large excess of inert gas (argon N60 or xenon N48) on a CsI window cooled to $10 \mathrm{~K}$ (argon) or $20 \mathrm{~K}$ (xenon). The temperature of sublimation was $c a .75^{\circ} \mathrm{C}$. At this temperature, sublimation occurs without degradation, as indicated by the absence of any band in the spectrum that could be ascribed to products of decomposition (e.g., $\mathrm{HNCO}, \mathrm{CO}^{19}$ ). After deposition of the compound, annealing experiments were performed until a temperature of $40 \mathrm{~K}(\mathrm{Ar})$ or $60 \mathrm{~K}(\mathrm{Xe})$ was attained (in steps of $3 \mathrm{~K}$ ). In the annealing experiments, the temperature was controlled and measured by a diode temperature sensor, connected to a Scientific Instruments digital temperature controller (model 9659), to within $1 \mathrm{~K}$. The low temperature solid films of PA and NA were prepared in a way similar to that used to obtain the matrices, but in this case only vapours of the compound were deposited onto the CsI substrate of the cryostat, which was kept at $10 \mathrm{~K}$ during deposition. The room temperature infrared spectra of the crystalline PA and NA were obtained in $\mathrm{KBr}$ pellets. In both matrix-isolation and neat solid state studies the infrared spectra were collected, with $0.5 \mathrm{~cm}^{-1}$ spectral resolution, on a Mattson (Infinity 60AR Series) or Thermo Nicolet Nexus 670 FTIR spectrometer, equipped with deuterated triglycine sulfate (DTGS) detectors and $\mathrm{Ge} / \mathrm{KBr}$ or "solid substrate" (silicon) $)^{28}$ beamsplitters.

PA and NA and their dimers were studied by pulsed supersonic jet FTIR spectroscopy. ${ }^{29}$ The expansion from a slit nozzle of $10 \times 0.5 \mathrm{~mm}^{2}$ cross-section (heatable design ${ }^{30}$ ) was probed in direct absorption by a focussed FTIR beam. Reference spectra without expansion ( $I_{\mathrm{o}}$, vacuum) were collected immediately before the gas pulse and used to determine the absorbance, $\ln \left(I_{\mathrm{o}} / I\right)$, from the expansion spectra $(I)$. The buildup of background pressure in the pulsed jet experiments was limited by a $4 \mathrm{~m}^{3}$ stainless steel vacuum chamber followed by a $250 \mathrm{~m}^{3} \mathrm{~h}^{-1}$ pumping system and by up to $40 \mathrm{~s}$ pumping intervals between pulses. Typically, data from one hundred to several hundred gas pulses were co-added for the IR spectra shown in this work. Gas pulses were controlled by magnetic valves. For details, see the cited ref. 29 and 30. The FTIR 
signal (Bruker IFS 66v) was detected by a large area InSb or MCT detector equipped with an appropriate optical filter. The spectral resolution was $2 \mathrm{~cm}^{-1}$. PA and NA were adsorbed on a dried molecular sieve and seeded into the He $(99.996 \%$, Air Liquide) carrier gas by flowing the gas at 1-2 bar through a heated (to $100{ }^{\circ} \mathrm{C}$ for PA and to $135{ }^{\circ} \mathrm{C}$ for $\mathrm{NA}$ ) reservoir containing the molecular sieve.

\section{Computational methodology}

The quantum chemical calculations were performed with the Gaussian 03 program $^{31}$ at the DFT/(B3LYP $)^{32-34}$ and MP2 ${ }^{35}$ levels of theory, using the $6-311++\mathrm{G}(\mathrm{d}, \mathrm{p})$ basis set. ${ }^{36}$ The DFT(B3LYP)/6-311++G(d,p) calculated vibrational frequencies were scaled down by 0.960 in the $\mathrm{N}-\mathrm{H}$ and $\mathrm{C}-\mathrm{H}$ stretching regions and 0.988 for all the remaining spectral regions, and used to help interpretation of the experimentally observed spectra. They should be reliable for monomers and hydrogen-bonded dimers, as long as no dispersion interaction between the aromatic rings is involved. Normal coordinate analysis was undertaken in the internal coordinates space as described by Schachtschneider, ${ }^{37}$ using the noncommercial program BALGA and home made (by L.L.) programs.

\section{Results and discussion}

\section{Matrix isolation infrared spectra of nicotinamide monomer}

The interpretation of the matrix isolation infrared spectroscopy data, obtained in the present study for NA, received support from theoretical calculations on the monomeric forms of the compound, undertaken at the B3LYP and MP2 levels of theory, with the $6-311++\mathrm{G}(\mathrm{d}, \mathrm{p})$ basis set. The present MP2/6-311 + $+\mathrm{G}(\mathrm{d}, \mathrm{p})$ calculations correspond to the highest theoretical level applied to the study of this compound hitherto. The main reason for additional calculations at the B3LYP/6-311 + + G(d,p) level of approximation is their wellknown good and economical performance in calculating vibrational spectra. ${ }^{38,39}$ The theoretical structural results on monomeric NA and potential energy profiles for conformational isomerization are provided in the ESI (Fig. S1 and S2 and Table $\mathrm{S} 1)+\dagger$ The results of the present calculations follow the general trend of previous studies on this molecule undertaken at comparable theoretical approximation levels. ${ }^{11,16}$

At the MP2/6-311++G(d,p) level, the $\mathrm{C}_{2}-\mathrm{C}_{3}-\mathrm{C}_{11}-\mathrm{O}_{15}$ dihedral angle was found to be equal to 148.6 and $24.0^{\circ}$ in $E$ and $Z$ forms (see Scheme 2), respectively, in fairly good agreement with the experimentally available data. ${ }^{11,12}$ The $E$ conformer is predicted to be more stable than the $Z$ form by $3.0 \mathrm{~kJ} \mathrm{~mol}^{-1}$ (zero-point energy corrected electronic energy difference) with the $Z \rightarrow E$ barrier for conformational isomerization being $8.2 \mathrm{~kJ} \mathrm{~mol}^{-1}$. The calculated Gibbs energy difference between the two conformers at the temperature of sublimation of the compound in the matrix isolation experiments $\left(\mathrm{ca} .75^{\circ} \mathrm{C}\right)$ was estimated by the calculations as 2.5 or $4.7 \mathrm{~kJ} \mathrm{~mol}^{-1}$, at the MP2 and DFT/B3LYP level of theory, respectively (average value: $3.6 \mathrm{~kJ} \mathrm{~mol}^{-1}$ ), being considerably lower than that reported in the microwave spectroscopy work

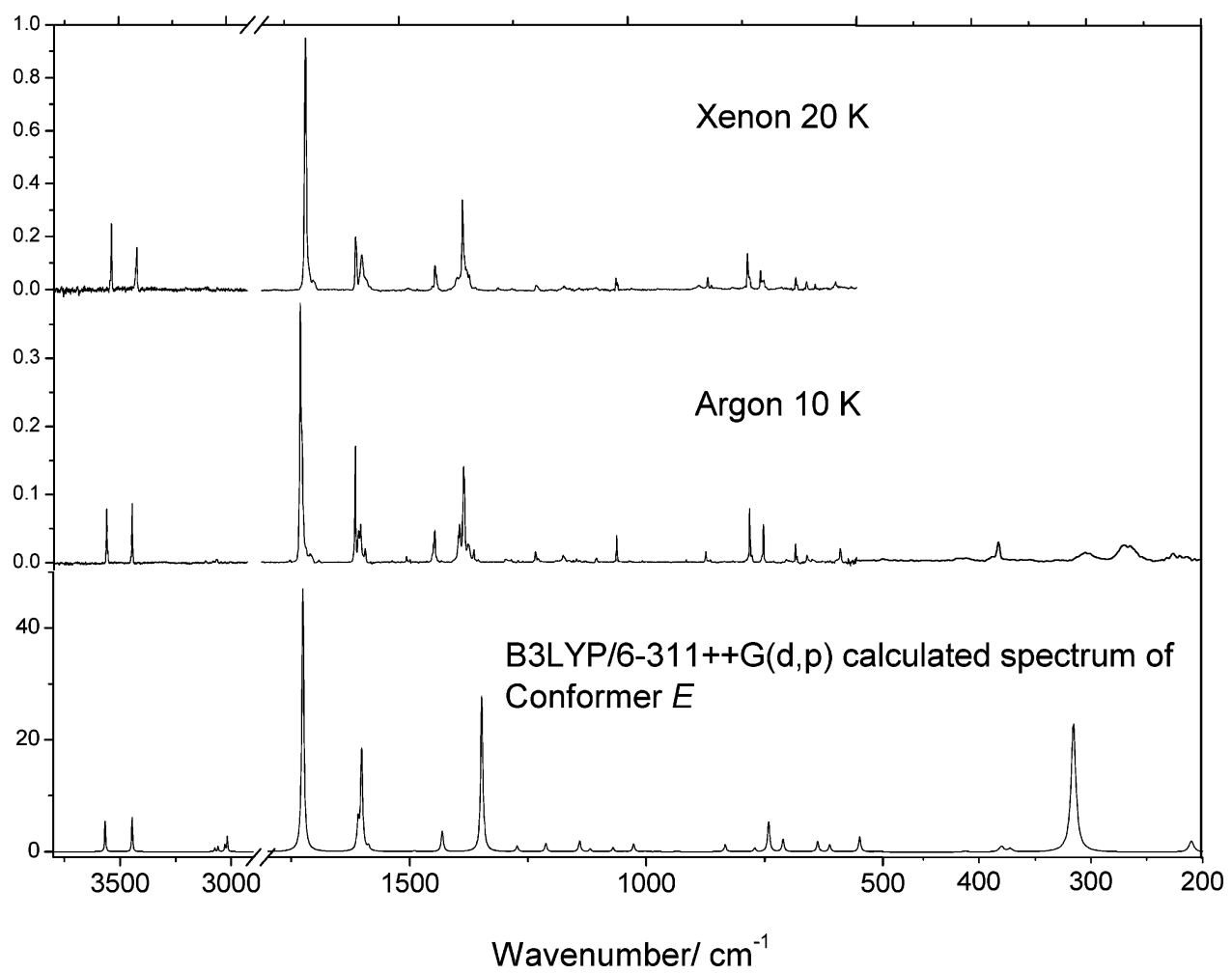

Fig. 1 Experimental infrared spectra of nicotinamide in argon and xenon matrices and B3LYP/6-311+ $+\mathrm{G}(\mathrm{d}, \mathrm{p})$ calculated spectrum for the $E$ isomer of this molecule in the $3800-200 \mathrm{~cm}^{-1}$ spectral range. The calculated spectrum was simulated using Lorentzian functions with halfbandwidth of $5 \mathrm{~cm}^{-1}$, frequencies being scaled by 0.960 in the high frequency range and 0.988 below $1800 \mathrm{~cm}^{-1}$. Ordinate scales of the experimental spectra are in decadic absorbance; that of the calculated spectrum is in arbitrary units. 
(6.6-12.6 $\left.\mathrm{kJ} \mathrm{mol}^{-112}\right)$ but in agreement with the clear dominance of the $E$ conformer in the gas phase. The dipole moments of the two conformers were found to be very different. At the MP2 $/ 6-311++\mathrm{G}(\mathrm{d}, \mathrm{p})$ level of theory, the dipole moments in the $E$ and $Z$ forms are predicted to be 2.6 and 5.2 Debye, respectively, which can be compared with those derived from microwave spectroscopy (1.2 and 4.5 Debye $^{12}$ ). On the whole, taking into consideration the previous experimental data for the NA in the gas phase and the results of the present calculations, in particular the low energy difference between the two conformers and the relatively large energy barrier separating them, it could be anticipated that both $E$ and $Z$ conformers should be trapped in the cryogenic matrices.
Nicotinamide has 39 fundamental vibrations, all infrared active for both $E$ and $Z$ conformers. Table S2 (ESI $\dagger$ ) displays the definition of symmetry coordinates used in the normal coordinate analysis undertaken in this study. The B3LYP/ 6-311 ++ G(d,p) calculated spectra for both conformers of NA and potential energy distributions (PED) are given in Tables S3-S4. $\dagger$ Fig. 1 shows the infrared spectra of NA isolated in argon (10 K; 4000-200 $\mathrm{cm}^{-1}$ spectral range) and xenon $(20 \mathrm{~K}$; $4000-500 \mathrm{~cm}^{-1}$ region) matrices. As seen in the figure, these spectra agree well with the calculated spectrum of the $E$ conformer. The band assignments are given in Table 1.

The identification of the minor conformer $(Z)$ in the matrices was not easy. The main difficulty of this task resulted from the great similarity between the spectra of the two conformers

Table 1 Observed wavenumbers for the $E$ form of nicotinamide monomer in argon and xenon matrices with band assignments. B3LYP/ $6-311++\mathrm{G}(\mathrm{d}, \mathrm{p})$ calculated (scaled) wavenumbers and intensities are also given ${ }^{a}$

\begin{tabular}{|c|c|c|c|c|}
\hline Approximate description & Calculated wavenumber & Calculated intensity & Observed $^{b} \operatorname{Ar}(10 \mathrm{~K})$ & Observed Xe (20 K) \\
\hline$\nu\left(\mathrm{NH}_{2}\right)$ as & 3568 & 43 & 3555 & 3533 \\
\hline$\nu\left(\mathrm{NH}_{2}\right) \mathrm{s}$ & 3446 & 48 & 3437 & 3415 \\
\hline$\nu(\mathrm{C}-\mathrm{H})^{\prime \prime} 1$ & 3074 & 5 & 3095 & n.o. \\
\hline$\nu(\mathrm{C}-\mathrm{H})^{\prime \prime} 2$ & 3059 & 8 & 3075 & n.o. \\
\hline$\nu(\mathrm{C}-\mathrm{H})^{\prime} 1$ & 3029 & 9 & $3062 / 3055$ & n.o. \\
\hline$\nu(\mathrm{C}-\mathrm{H})^{\prime} 2$ & 3018 & 21 & 3042 & n.o. \\
\hline$\nu(\mathrm{C}=\mathrm{O})$ & 1726 & 369 & $1715 / 1711$ & 1704 \\
\hline$v \operatorname{ring} 1$ & 1609 & 39 & $1588 / 1583^{c}$ & $1581^{c}$ \\
\hline$\delta\left(\mathrm{NH}_{2}\right)$ & 1601 & 142 & $1595^{c}$ & $1594^{c}$ \\
\hline$\nu$ ring 5 & 1587 & 6 & 1573 & 1563 \\
\hline$\delta(\mathrm{C}-\mathrm{H})^{\prime} 2$ & 1490 & 2 & 1483 & 1484 \\
\hline$\delta(\mathrm{C}-\mathrm{H})^{\prime} 1$ & 1431 & 29 & $1424 / 1421$ & 1421 \\
\hline$\nu(\mathrm{C}-\mathrm{N})$ & 1348 & 216 & $1370 / 1367 / 1358 / 1347 / 1336^{d}$ & $1372 / 1361 / 1352 / 1346^{d}$ \\
\hline$\delta(\mathrm{C}-\mathrm{H})^{\prime \prime} 1$ & 1343 & 11 & & \\
\hline$v \operatorname{ring} 4$ & 1273 & 8 & $1267 / 1265$ & 1283 \\
\hline$\nu \operatorname{ring} 6$ & 1212 & 11 & 1201 & 1200 \\
\hline$\delta(\mathrm{C}-\mathrm{H})^{\prime \prime} 2$ & 1141 & 15 & 1141 & 1139 \\
\hline$\nu$ ring 2 & 1118 & 4 & 1111 & 1106 \\
\hline $\mathrm{w}\left(\mathrm{NH}_{2}\right)$ & 1070 & 6 & 1068 & 1069 \\
\hline$\nu \operatorname{ring} 3$ & 1046 & 1 & 1038 & 1036 \\
\hline$\delta$ ring 1 & 1027 & 10 & 1024 & $1026 / 1023$ \\
\hline$\gamma(\mathrm{C}-\mathrm{H})^{\prime} 2$ & 1002 & 1 & 993 & 992 \\
\hline$\gamma(\mathrm{C}-\mathrm{H})^{\prime \prime} 2$ & 976 & 1 & 968 & 968 \\
\hline$\gamma(\mathrm{C}-\mathrm{H})^{\prime \prime} 1$ & 935 & 1 & 935 & 935 \\
\hline$\gamma(\mathrm{C}-\mathrm{H})^{\prime} 1$ & 833 & 10 & 829 & 826 \\
\hline$\nu(\mathrm{C}-\mathrm{C})$ & 771 & 5 & 770 & 770 \\
\hline$\gamma(\mathrm{C}=\mathrm{O})$ & 741 & 42 & $733 / 728$ & $738 / 734$ \\
\hline$\tau$ ring 1 & 711 & 17 & 703 & $710 / 706 / 703$ \\
\hline$\delta(\mathrm{C}=\mathrm{O})$ & 638 & 14 & 633 & 633 \\
\hline$\delta$ ring 2 & 613 & 9 & 608 & 609 \\
\hline$\tau(\mathrm{C}-\mathrm{N})$ & 549 & 21 & $536 / 534$ & 546 \\
\hline$\delta(\mathrm{C}-\mathrm{C}-\mathrm{N})$ & 491 & 2 & 477 & n.o. \\
\hline$\tau$ ring 3 & 412 & 1 & $409 / 404$ & n.o. \\
\hline$\tau$ ring 2 & 380 & 7 & 381 & n.i. \\
\hline$\delta$ ring 3 & 372 & 4 & 376 & n.i. \\
\hline$\gamma\left(\mathrm{NH}_{2}\right)$ & 316 & 176 & $301 / 266^{e}$ & n.i. \\
\hline $\mathrm{w}(\mathrm{C}-\mathrm{C})$ & 210 & 15 & 224 & n.i. \\
\hline$\gamma(\mathrm{C}-\mathrm{C})$ & 149 & 3 & n.i. & n.i. \\
\hline$\tau(\mathrm{C}-\mathrm{C})$ & 54 & 14 & n.i. & n.i. \\
\hline
\end{tabular}

${ }^{a}$ Wavenumbers in $\mathrm{cm}^{-1}$, calculated intensities in $\mathrm{km} \mathrm{mol}^{-1} . \nu$, bond stretching, $\delta$, bending, $\gamma$, rocking, $\tau$, torsion, w, wagging, tw, twisting, $\mathrm{s}$, symmetric, as, antisymmetric, n.o. not observed, n.i. not investigated. ${ }^{b}$ Observed bands tentatively assigned to the $Z$ conformer occur at the following wavenumbers: $3551,3432,1718,1357,1349,1195,819$, and $630 \mathrm{~cm}^{-1}$; the band at $376 \mathrm{~cm}^{-1}$ may also contain an important contribution from the $\delta$ ring 3 and $\gamma\left(\mathrm{NH}_{2}\right)$ modes of the $Z$ form. ${ }^{c}$ The proposed assignments of the $v$ ring 1 and $\delta\left(\mathrm{NH}_{2}\right)$ modes are based on the relative intensity of the bands; on the other hand, taking the predicted relative wavenumbers of the bands ascribable to these modes into account, an alternative assignment would be the opposite one. ${ }^{d}$ This is a complex spectral region where the $\nu(\mathrm{C}-\mathrm{N})$ and $\delta(\mathrm{C}-\mathrm{H})^{\prime \prime} 1$ fundamentals are involved in multiple Fermi resonances with different combination tones: $\tau$ ring $1+\delta(\mathrm{C}=\mathrm{O}) ; \gamma(\mathrm{C}=\mathrm{O})+\delta(\mathrm{C}=\mathrm{O}) .{ }^{e}$ Fermi resonance with $2 \gamma(\mathrm{C}-\mathrm{C})$. 
(see Tables S3 and S4 and Fig. S3). $\dagger$ Practically all the bands due to the $Z$ form were then hidden underneath those belonging to the most abundant $E$ isomer. Moreover, the barrier for the $Z \rightarrow E$ conformational isomerization $\left(8.2 \mathrm{~kJ} \mathrm{~mol}^{-1}\right)$ is high enough to make this reaction unaccessible upon annealing of the argon matrices, since at the temperature by which the isomerization should start ( $c a .35 \mathrm{~K}$, according to the Barnes relationship ${ }^{40}$ ), extensive aggregation did already occur, introducing an additional complication in the analysis of the results. On the other hand, in a xenon matrix the working range of temperatures is wider. Temperature variation experiments were also undertaken in this medium. Unfortunately, for the xenon matrices, the spectral bands are in general broader than those recorded for the species isolated in solid argon. This circumstance constituted a serious drawback, taking into account the great similarity of the spectra of the two conformers. Nevertheless, there is a region of the spectrum where the calculations predict non-overlapping bands of the two isomers (at 833 and $823 \mathrm{~cm}^{-1}$, respectively, for $E$ and $Z$ conformers, $\Delta \nu=10 \mathrm{~cm}^{-1}$ ) and having sufficient intensity to be clearly observable (in all other accessible spectral regions the bands nearly coincide or have too low intensity to allow the observation of the band of the less populated conformer). The spectral region (around $820 \mathrm{~cm}^{-1}$ ) where the $\gamma(\mathrm{C}-\mathrm{H})^{\prime} 1$ bands due to the $E$ and $Z$ conformers appear as well-separated features is presented in Fig. 2. This figure summarizes also the results of the temperature variation experiments undertaken for nicotinamide in the xenon matrix. At the deposition temperature $(20 \mathrm{~K}$; thick solid line) absorptions due to $E\left(826 \mathrm{~cm}^{-1}\right)$ and $Z\left(818 \mathrm{~cm}^{-1}\right)$ conformers were observed. At $38 \mathrm{~K}$ (thin solid line), partial conversion of the less stable $Z$ conformer to the $E$ form is

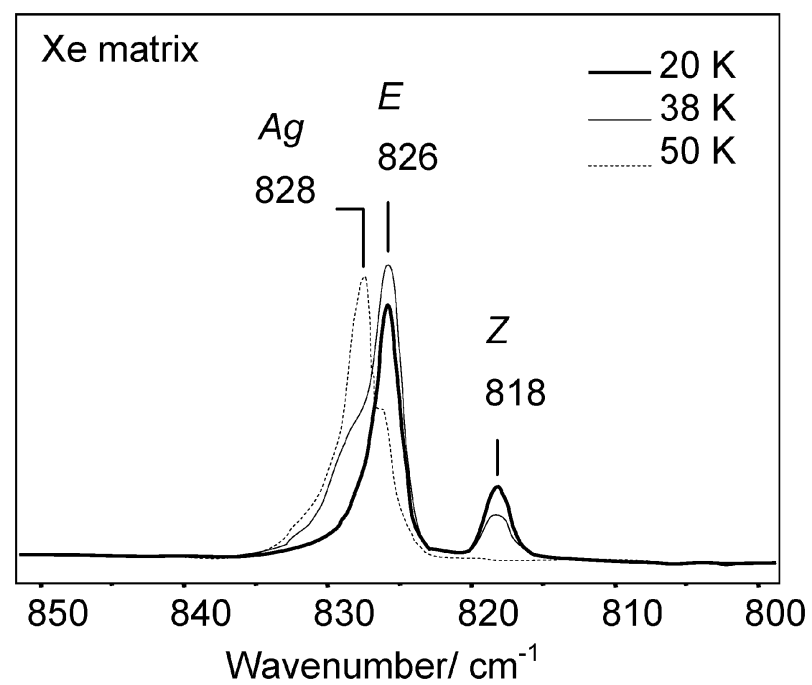

Fig. $2 \gamma(\mathrm{C}-\mathrm{H})^{\prime} 1$ spectral region of the infrared spectra of nicotinamide in a xenon matrix at different temperatures, during the temperature variation experiments. At the deposition temperature $(20 \mathrm{~K}$; thick solid line) absorptions due to $E\left(826 \mathrm{~cm}^{-1}\right)$ and $Z\left(818 \mathrm{~cm}^{-1}\right)$ conformers were observed; at $38 \mathrm{~K}$ (thin solid line), partial conversion of the less stable $Z$ conformer to the $E$ form is clearly visible, while a band due to aggregates $\left(828 \mathrm{~cm}^{-1}\right)$ has also already started to grow; at $50 \mathrm{~K}$, the band due to the $Z$ conformer has completely disappeared from the spectrum, while that due to aggregates dominates. clearly noticeable, as seen by the increase of the band due to the $E$ form and decrease of that ascribed to the $Z$ form (at this temperature, a band due to aggregates, at $828 \mathrm{~cm}^{-1}$, has also already started to grow). At $50 \mathrm{~K}$, the band due to the $E$ conformer can still be observed as a shoulder of the band of the aggregated species, while that of the $Z$ conformer has completely disappeared from the spectrum.

In the $\gamma(\mathrm{C}-\mathrm{H})^{\prime} 1$ spectral region of the spectra obtained in argon matrices, the bands ascribed to the $E$ and $Z$ conformers are also observable (at 829 and $819 \mathrm{~cm}^{-1}$, respectively). Indeed, in consonance with the results obtained for a xenon matrix, the band due to the $Z$ conformer disappears when the temperature of the argon matrix exceeds $c a .35 \mathrm{~K}$. Similar behaviour was observed for other bands in the spectra of argon matrices. These bands were also tentatively assigned to $\begin{array}{lllllll}\text { conformer } Z: & 3551 & {\left[\nu\left(\mathrm{NH}_{2}\right) \mathrm{as}\right],} & 3432 & {\left[\nu\left(\mathrm{NH}_{2}\right) \mathrm{s}\right],} & 1718\end{array}$ $[\nu(\mathrm{C}=\mathrm{O})], 1357\left[\delta(\mathrm{C}-\mathrm{H})^{\prime} 1\right], 1349[\nu(\mathrm{C}-\mathrm{N})], 1195\left[\gamma(\mathrm{C}-\mathrm{H})^{\prime} 2\right]$, and $630[\delta$ ring 3$] \mathrm{cm}^{-1}$; the band at $376 \mathrm{~cm}^{-1}$ shall also contain an important contribution from the $\delta(\mathrm{C}=\mathrm{O})$ and $\gamma\left(\mathrm{NH}_{2}\right)$ modes of the $Z$ form.

From the relative absorbances of the $\gamma(\mathrm{C}-\mathrm{H})^{\prime} 1$ bands of the two conformers observed in the as-deposited matrices (both argon and xenon matrices), weighted by the corresponding calculated intensities, the population of the two conformers in the gas phase at $75{ }^{\circ} \mathrm{C}$ (the sublimation temperature of the compound in the performed experiments) could be estimated. The results obtained using the data extracted from both argon and xenon matrices agree with each other and yield populations for $E$ and $Z$ conformers equal to $80 \%$ and $20 \%$, respectively. This implies that the energy difference between the conformers is $c a$. $4.0 \mathrm{~kJ} \mathrm{~mol}^{-1}$. Interestingly, this energy difference coincides almost exactly with the average $\left(3.6 \mathrm{~kJ} \mathrm{~mol}^{-1}\right)$ of the values theoretically predicted using the DFT and MP2 methods. The free energy difference between $E$ and $Z$ conformers of nicotinamide obtained above, differs from the previous assessment. ${ }^{12}$

\section{Infrared spectra of nicotinamide and picolinamide monomers and dimers in the supersonic jet}

For nicotinamide and picolinamide in the jet expansion (using $\mathrm{He}$ as the carrier gas), the infrared spectra were recorded in the $3600-3000 \mathrm{~cm}^{-1}$ and $2000-1000 \mathrm{~cm}^{-1}$ spectral regions. As described in detail below, these spectra show features ascribable to the monomer as well as to the dimer of the compounds. In order to facilitate interpretation of the experimental spectra, calculations were carried out for the dimeric structures of both PA and NA. The calculations were performed for different possible structures of the dimer, built of the monomers in their most stable $(E)$ form (see Fig. 3).

For both compounds, the electronic energy of the centrosymmetric dimer (dimer 1) was found to be considerably lower than any of the other dimeric structures. The dimerization energy to form the centrosymmetric dimer in PA was found to be $c a .-45 \mathrm{~kJ} \mathrm{~mol}^{-1}$, whereas for the centrosymmetric dimer of NA the analogous value is $c a .-49 \mathrm{~kJ} \mathrm{~mol}^{-1}$. This indicates that the $\mathrm{NH}$... O hydrogen bonds are stronger in this latter species. In agreement with the structural results, the calculated frequency of the $\mathrm{N}-\mathrm{H}$ stretching mode associated with the 
NA
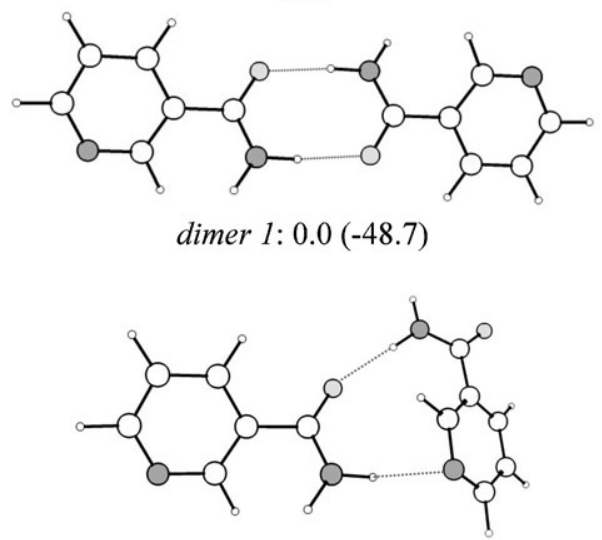

dimer 2: $15.1(-33.6)$

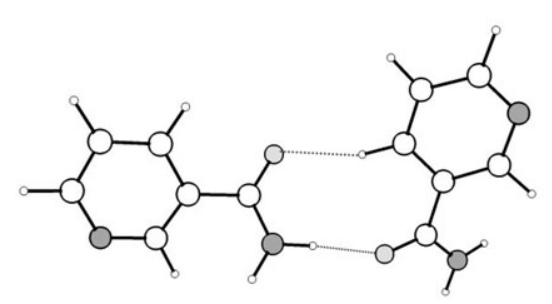

dimer 3: $19.1(-29.6)$

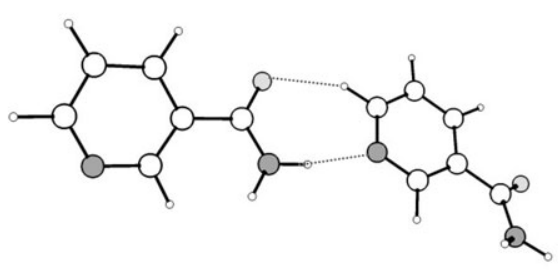

dimer 4: $22.8(-25.9)$
PA

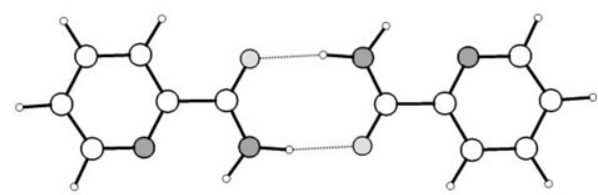

dimer 1: $0.0(-44.7)$

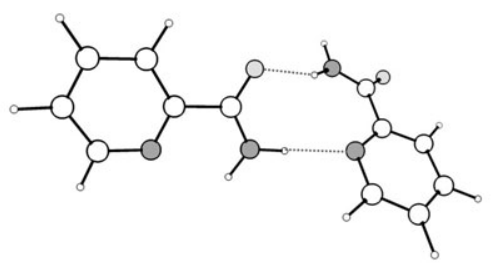

dimer 2: $21.3(-23.4)$

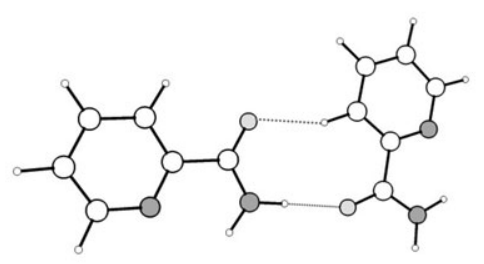

dimer 3: $16.5(-28.2)$

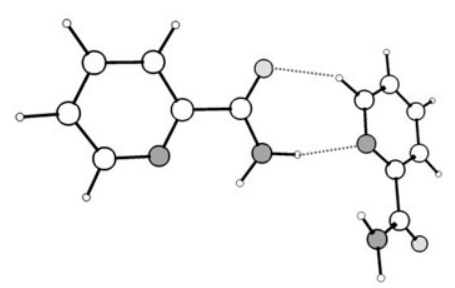

dimer 4: $29.7(-15.0)$

Fig. 3 DFT(B3LYP) $/ 6-311++$ G(d,p) calculated structures for the most stable dimers of nicotinamide and picolinamide based on the most stable $E$ monomeric structure. Relative energies of the dimers and dimerization energies [calculated as $E$ (dimer)-2x $E$ (monomer); in parentheses] are given in $\mathrm{kJ} \mathrm{mol}^{-1}$.

intermolecular H-bonds is lower in NA than in PA (3204 vs. $3225 \mathrm{~cm}^{-1}$ ) and the corresponding IR intensity larger (2566 vs. $2261 \mathrm{~km} \mathrm{~mol}^{-1}$ ). The weaker intermolecular H-bonds in the centrosymmetric dimer of PA (compared to NA) can be easily explained taking into account the influence of the intramolecular H-bond between the amide group and the ring nitrogen atom in this molecule, which withdraws electronic charge from the NH...O intermolecular H-bond. Thus, we have a competitive hydrogen bond situation.

The calculated spectroscopic data (full set of vibrational frequencies, IR intensities and results of normal coordinate analysis) for the relevant dimers of PA and NA are provided in the ESI (Tables S5-S10). $\dagger$

Fig. 4 compares the supersonic jet spectra of PA (top) and NA (bottom) in the $\mathrm{N}-\mathrm{H}$ stretching range, which is particularly sensitive to aggregation. The monomer bands marked $\mathrm{M}$ are in good agreement with the matrix isolation data ( $\mathrm{Ar}$ matrix; see also ref. 19 in the case of PA), with fairly systematic matrix-induced red-shifts between 11 and $13 \mathrm{~cm}^{-1}$. Only the antisymmetric N-H stretching band of PA deviates slightly more, due to significant site splitting. It is the strongest of the $\mathrm{N}-\mathrm{H}$ stretching bands of the monomer, also according to the calculations. This reflects some degree of intramolecular hydrogen bonding to the pyridine nitrogen. The free $\mathrm{N}-\mathrm{H}$ stretching mode in the dimers which correlates with this antisymmetric monomer $\mathrm{N}-\mathrm{H}$ stretching fundamental also shows characteristic differences between PA and NA. In PA, it is further red-shifted than in NA. This is also a consequence of its internal hydrogen bond involvement, which matches the theoretical prediction. The most characteristic difference is found for the intermolecularly $\mathrm{H}$-bonded $\mathrm{N}-\mathrm{H}$ stretching mode between 3100 and $3200 \mathrm{~cm}^{-1}$. Its red shift, intensity and sharpness are all more pronounced in NA than in PA. While the former two quantities are known to correlate with each other and to signal a stronger hydrogen bond involvement, the latter category is usually anticorrelated. Stronger hydrogen bonds tend to lead to broader, often Fermiresonance modulated bands even at cryogenic temperatures. 


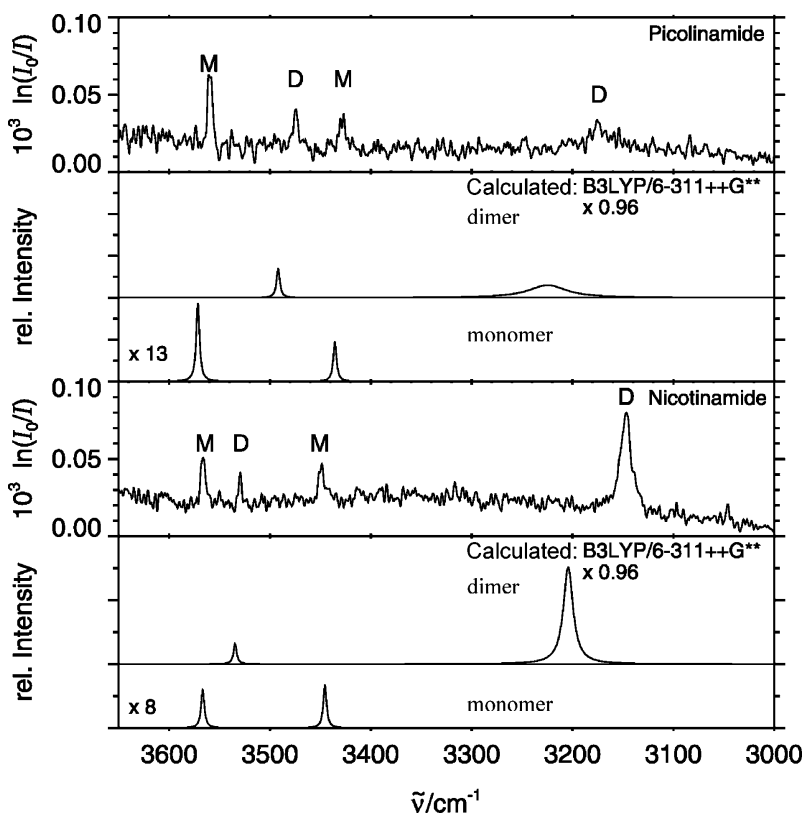

Fig. 4 N-H stretching range of the jet spectra of PA (top, sample at $100{ }^{\circ} \mathrm{C}$ expanded in 1.4 bar $\mathrm{He}$ ) and NA (bottom, sample at $135{ }^{\circ} \mathrm{C}$ expanded in 1.4 bar $\mathrm{He}$ ) compared to scaled harmonic B3LYP calculations for the amide monomers and dimers. The monomer and free dimer bands are modelled by Lorentzian profiles with a full width at half maximum of $4 \mathrm{~cm}^{-1}$, whereas the $\mathrm{H}$-bonded $\mathrm{N}-\mathrm{H}$ bands are qualitatively approximated by widths of 12 (NA) and 48 (PA) $\mathrm{cm}^{-1}$. The intensities of the bands in the IR spectra simulated for monomers are scaled by a factor of 13 (8) relative to the dimer bands, reflecting an approximate dimer number fraction of $7 \%(11 \%)$ in the jet expansion zone for PA(NA). Note the $10^{-4}$ napierian absorbance scale in the jet spectra, which underlines the low density of molecular absorbers in the jet experiment.

Instead, the stronger intermolecular hydrogen bond in NA leads to a $4 \pm 2$ times sharper band, according to the Lorentzian simulation of the theoretical spectrum. At the present signal-to-noise ratio, it is difficult to exclude structure in the broad PA dimer band profile. Independent of the existence of such structure, ${ }^{41}$ the PA dimer band reflects an intramolecular vibrational relaxation rate which is about 4 times faster than that in the corresponding NA band. This rapid energy flow on the $0.1 \mathrm{ps}$ time scale must be related to the weak intramolecular hydrogen bond which is specific to PA. Vibrational energy deposited in the red-shifted $\mathrm{N}-\mathrm{H}$ stretching mode can flow efficiently into the framework modes of the molecule, probably via $\mathrm{N}-\mathrm{H}$ bending overtone states. A similar phenomenon of resonance tuning can be observed in pyrazole clusters. ${ }^{42}$ What makes the present case special is the unambiguous effect of a weak intramolecular $\mathrm{N}-\mathrm{H} \cdots \mathrm{N}$ hydrogen bond in switching on this additional relaxation channel. Significant differences in inhomogeneous contributions to the observed dimer band widths between PA and NA can be ruled out by the comparison of the two very similar systems under identical expansion conditions.

It is also useful to compare the present $\mathrm{N}-\mathrm{H}$ stretching spectra to those of the non-aromatic parent compound acetamide (AA) and its dimer. ${ }^{27}$ The monomer transitions of NA are blue-shifted by 2 and $9 \mathrm{~cm}^{-1}$ relative to those of AA, whereas the $\mathrm{N}-\mathrm{H}$ stretches of PA are red-shifted by 4 and $13 \mathrm{~cm}^{-1}$ due to the intramolecular $\mathrm{H}$-bond. For the free $\mathrm{N}-\mathrm{H}$ in the dimer, there is a blue shift of $3 \mathrm{~cm}^{-1}$ in NA and a red shift of $53 \mathrm{~cm}^{-1}$ in PA, relative to AA. This clearly shows that the intramolecular hydrogen bond effect is amplified in the dimer. On the other hand, there is a red shift of $20 \mathrm{~cm}^{-1}$ in NA and a blue shift of $9 \mathrm{~cm}^{-1}$ in PA for the intermolecularly $\mathrm{H}$-bonded $\mathrm{N}-\mathrm{H}$, i.e. just the opposite behaviour relative to AA. This underlines the substantial hydrogen bond competition between the two $\mathrm{N}-\mathrm{H}$ stretching modes in the amide group.

Besides the $3600-3000 \mathrm{~cm}^{-1}$ range, Fig. 5 and 6 show the $2000-1000 \mathrm{~cm}^{-1}$ regions of the infrared spectra of PA and NA, respectively, in the supersonic jet. These spectra are compared in the figures with those obtained for the monomeric species in an argon matrix $(10 \mathrm{~K})$ as well as with those calculated for the monomer ( $E$ form) and for the centrosymmetric dimer (dimer 1). The signal-to-noise ratio in the jet spectra is limited, allowing only for a confirmation of the strongest monomer bands ( $E$ isomer) in the fingerprint region. However, the centrosymmetric dimer $\mathrm{C}=\mathrm{O}$ stretching band reflects more directly the extent of aggregation in the jet, which is only on the order of $10 \%$. The assignments for the spectra of the compounds in the jet are given in Tables 2 and 3. The dimerization red-shift of the $\mathrm{C}=\mathrm{O}$ stretching mode and the blue-shift of the $\mathrm{C}-\mathrm{N}$ stretching mode are $50 \%$ larger in NA than in PA, again reflecting the significantly stronger intermolecular $\mathrm{N}-\mathrm{H} \cdots \mathrm{O}=\mathrm{C}$ hydrogen bond in NA dimer.

\section{Infrared spectra of nicotinamide and picolinamide in the low temperature glass and crystalline states}

According to previous structural studies ${ }^{22-24}$ the basic units in the crystals of NA and PA are different. The NA crystal was found to be monoclinic, $P 2_{1} / c(a=3.98, b=15.63$, $c=9.44 \AA, \beta=99.0^{\circ}$, at $295 \mathrm{~K}$ ), with 4 molecules per unit cell. NA molecules assume the $E$ conformation, with an angle between the pyridine and carboxamide moieties of $22^{\circ}$ and the amide group twisted by $c a .13^{\circ}$. They are stacked along the $a$ axis with the planar pyridine rings in contact (with a plane-to-plane distance of $c a$. $3.5 \AA$ ). ${ }^{23}$ Two intermolecular hydrogen bonds are established by the amide group, one with the oxygen atom of a neighbouring molecule and the second one with the pyridine nitrogen atom of a third molecule (at $295 \mathrm{~K}$ the $\mathrm{H}_{2} \mathrm{~N} \cdots \mathrm{O}^{\prime}$ and $\mathrm{H}_{2} \mathrm{~N} \cdots \mathrm{N}_{(\text {pyr) }}$ " distances were found to be 2.991 and $3.105 \AA$, respectively) ${ }^{23}$ (Scheme 3). In contrast, PA crystallizes in two modifications (both belonging to the monoclinic space group $P 2_{1} / a$, with 4 molecules in the unit cell). In one modification ( $\alpha$ phase; $a=16.42, b=7.11$, $c=5.19 \AA$, $\beta=100.2^{\circ}$ ), two PA molecules are joined together by a pair of $\mathrm{NH}$...O hydrogen bonds to form a centrosymmetric dimer, and such dimers are linked by a second set of $\mathrm{NH}$... O hydrogen bonds to make infinite chains that are parallel to each other; in this modification, the ring does not contribute to intermolecular hydrogen bonding and the adjacent chains are held together by van der Waals forces ${ }^{24}$ (see Scheme 3). The second crystalline modification of PA $\left(\beta\right.$ phase; $\left.a=20.04, b=11.32, c=5.36 \AA, \beta=98.6^{\circ}\right)$ exhibits a dimeric structure similar to that of the first one, but 

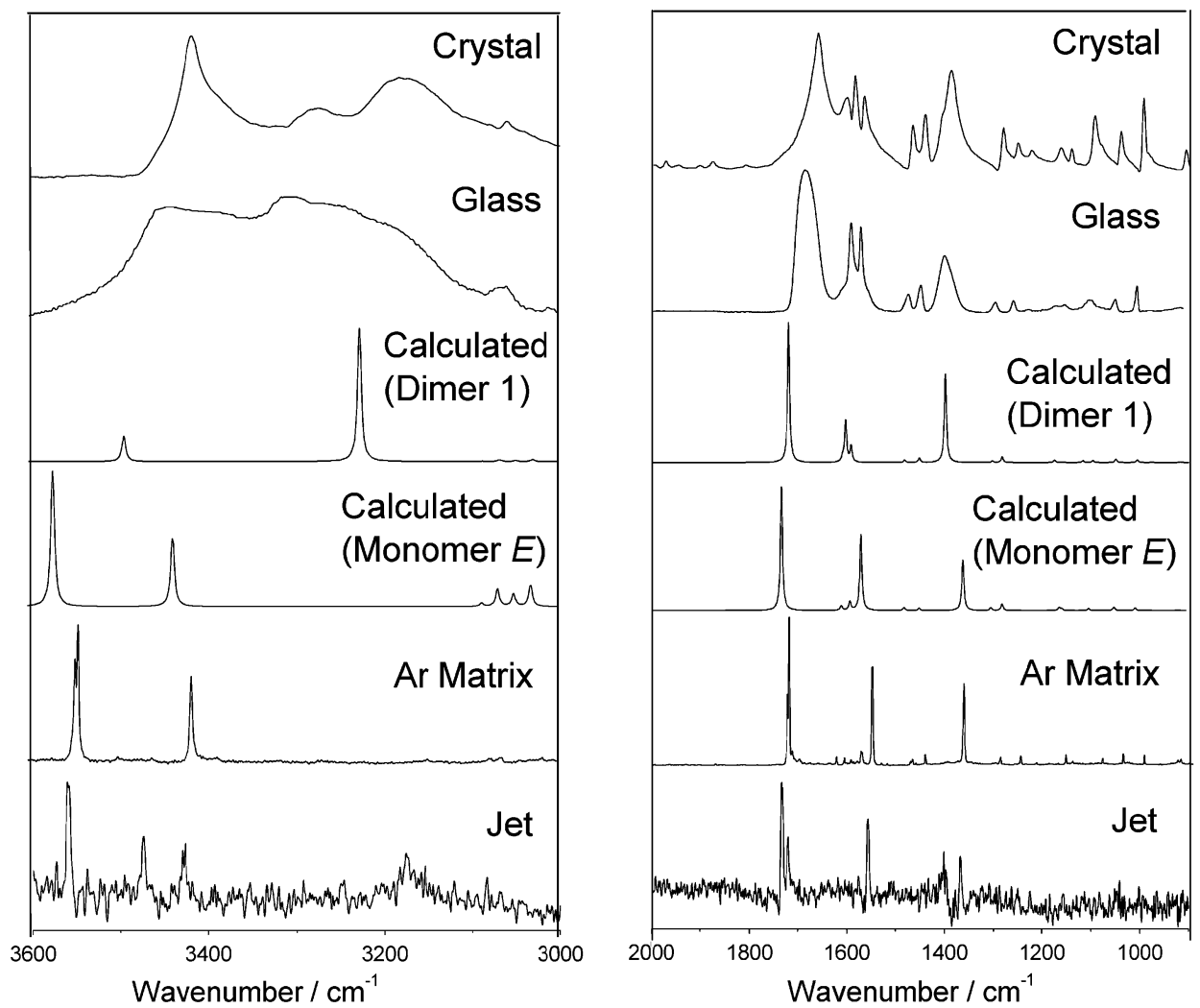

Fig. 5 Selected regions of the infrared spectra of picolinamide. From the bottom to the top: in the jet, in argon matrix (10 K), B3LYP/6$311++\mathrm{G}(\mathrm{d}, \mathrm{p})$ calculated for the monomer ( $E$ form; scaled by 0.960 in the high frequency region and by 0.988 in the low frequency region), calculated for the centrosymmetric dimer (dimer 1) using the same method and scalling factors as for the monomer, glassy state obtained by fast deposition of the vapour of the compound onto the cold tip of the cryostat $(10 \mathrm{~K})$ and room temperature crystalline state $\left(\mathrm{KBr}\right.$ pellet; $\left.25^{\circ} \mathrm{C}\right)$. Note that in the different spectra the intensity scales are different (in each spectral region each spectrum was normalized to the corresponding most intense band) so that comparison of intensities shown in the figure is only meaningful if it refers to the same spectrum and the same spectral region.

in this case the lateral linkage of the dimers involves the participation of an atom of the pyridine ring as $\mathrm{H}$-bond acceptor. ${ }^{24}$ In both PA crystalline modifications, the carboxamide group was found to be planar, with the angle between the pyridine and carboxamide moieties being equal to $19^{\circ}$ in the first crystalline modification. ${ }^{24}$ To the best of our knowledge, no data for this structural parameter in the second crystalline modification has been reported hitherto.

Fig. 5 and 6 present the $3600-3000 \mathrm{~cm}^{-1}$ and $2000-1000 \mathrm{~cm}^{-1}$ regions of the infrared spectra obtained for the glassy state resulting from fast deposition of the vapour of the compounds onto the cold tip of the cryostat $(10 \mathrm{~K})$ and for their room temperature crystalline state $\left(\mathrm{KBr}\right.$ pellet; $25{ }^{\circ} \mathrm{C}$; for $\mathrm{PA}$, $\alpha$-phase). The IR spectra of crystalline PA and NA correspond to the different networks of $\mathrm{H}$-bonds between molecules of the compounds present in a solid state. The systems of these bonds are shown in Scheme $3 .^{22-24}$ For NA, expansions of the $1800-1450 \mathrm{~cm}^{-1}$ and $1230-950 \mathrm{~cm}^{-1}$ spectral ranges are provided in Fig. 7 and 8, to allow a better comparison of the spectra. The proposed assignments for these spectra (in the full $4000-500 \mathrm{~cm}^{-1}$ range) are given in Tables S11 and S12 (ESI). $\dagger$

For both compounds, the spectrum of the glassy state is consistent with the prevalence of the $E$ monomer-based centrosymmetric dimer (dimer 1 ) in this phase, the observed spectra resembling very much those calculated for this dimer (see
Fig. 5-8). This seems to indicate that, upon landing onto the cold $(10 \mathrm{~K})$ surface of the substrate, the molecules of both compounds, free from any geometric constraint imposed by a specific crystalline periodic arrangement, tend to aggregate as the thermodynamically most stable dimeric structure. On the other hand, as already mentioned, in the crystalline phase the basic structural units are different for PA and NA, being the centrosymmetric dimer in the first case, ${ }^{24}$ and a motif based on an arrangement structurally related with dimer 2 in the latter, ${ }^{22,23}$ with intermolecular hydrogen bonds between the amide group of one molecule and the oxygen atom of the second molecule and between the amide group of this latter and the pyridine nitrogen atom of the first (see Scheme 3). It is important to point out here that the dimerization energy to form dimer 2 in NA $\left(c a .-34 \mathrm{~kJ} \mathrm{~mol}^{-1}\right)$ is predicted to be considerably larger than the corresponding value calculated for dimer 2 of PA $\left(c a .-23 \mathrm{~kJ} \mathrm{~mol}^{-1}\right)$. This fact is of crucial importance in determining the different behaviour upon crystallization of the two compounds. In particular, it is important for an explanation of the relevance of dimer 2 like structures in the crystal of NA and the irrelevance of such structures in the case of crystalline PA. The greater relative stability of the dimer 2 in NA results mainly from two different factors. Firstly, (as it was already discussed for the centrosymmetric dimers) the intermolecular $\mathrm{H}$-bonds in $\mathrm{PA}$ are 

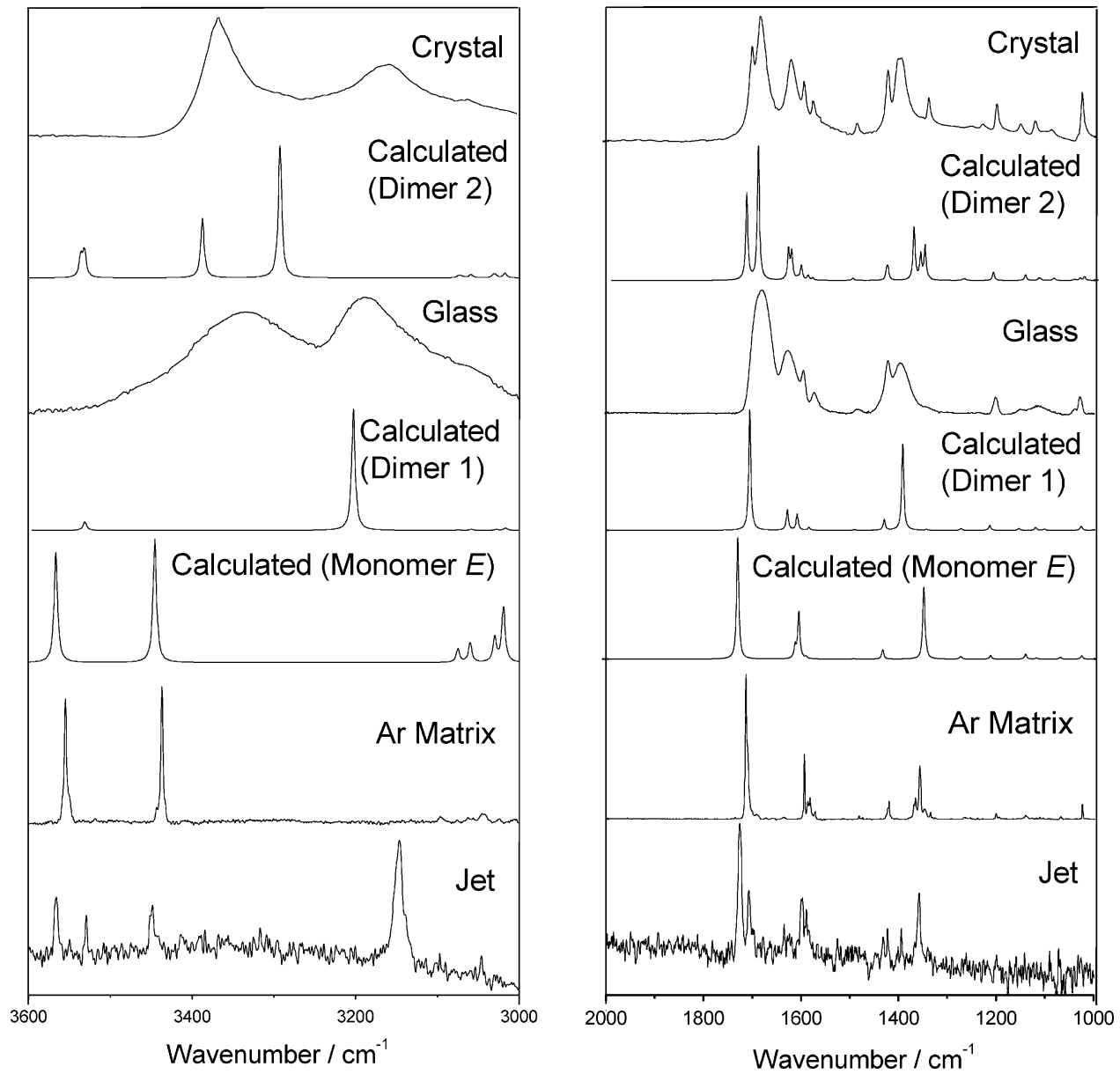

Fig. 6 Selected regions of the infrared spectra of nicotinamide. From the bottom to the top: in the jet, in argon matrix (10 K), B3LYP/6$311++\mathrm{G}(\mathrm{d}, \mathrm{p})$ calculated for the monomer ( $E$ form; scaled by 0.960 in the high frequency region and by 0.988 in the low frequency region), calculated for the centrosymmetric dimer (dimer 1) using the same method and scaling factors as for the monomer, glassy state obtained by fast deposition of the vapour of the compound onto the cold tip of the cryostat $(10 \mathrm{~K})$, calculated for dimer 2 and room temperature crystal-line state $\left(\mathrm{KBr}\right.$ pellet; $\left.25^{\circ} \mathrm{C}\right)$. Note that in the different spectra the intensity scales are different (in each spectral region each spectrum was normalized to the corresponding most intense band) so that comparison of intensities shown in the figure is only meaningful if it refers to the same spectrum and the same spectral region.

weakened by the simultaneous existence of the intramolecular $\mathrm{N}-\mathrm{H} \cdots \mathrm{N}_{\text {(ring) }}$ hydrogen bonds. Secondly, the greater conformational flexibility of NA around the C-C (amide-pyridyl) bond (which ultimately is also due to the absence of the intramolecular $\mathrm{NH} \cdots \mathrm{N}_{\text {(ring) }}$ interaction in this molecule) allows the molecule to adopt a much better geometry for establishment of the intermolecular hydrogen bonds in the dimer (see Fig. 3). The entropy of crystal formation is the other important factor, however, difficult to estimate theoretically. Of course, part of this entropy is connected with the conformational flexibility of the molecule.

As could be expected, when compared with the spectrum of the glassy state, the infrared spectrum of the crystalline phase of PA does not change very much (see Fig. 5), though the usual band narrowing in some spectral regions can be noticed (e.g., in the $3600-3000 \mathrm{~cm}^{-1}$ region). This result is in consonance with the considerably larger strength of the intermolecular $\mathrm{H}$-bonds within the dimeric units than those associated with the other interactions in the crystal, including the secondary hydrogen bonds that link the centrosymmetric dimers into endless parallel chains. ${ }^{24}$ On the other hand, the spectrum of crystalline NA differs considerably from that of the glassy state, while it shows a good agreement with the calculated spectra for dimer 2 (see Fig. 6-8). This is particularly relevant in the splitting of the carbonyl stretching region observed upon crystallization (Fig. 7), but it is also clearly shown in other spectral regions, like, for instance, the $1175-1075 \mathrm{~cm}^{-1}$ range (Fig. 8). Hence, the different behaviour of the two compounds upon crystallization is reflected in their vibrational signatures, which reveal the different types of basic structural units present in their crystalline phases. As pointed out above, this can be correlated with their molecular structures, in particular with the greater conformational mobility of NA compared with PA, due to the absence in the first molecule of the intramolecular $\mathrm{NH} \cdots \mathrm{N}_{\text {(ring) }}$ interaction, which dominates in the latter.

\section{Conclusion}

Matrix-isolation and supersonic jet techniques were applied to investigate monomers and dimers of nicotinamide and picolinamide by means of FTIR spectroscopy. The assignment was 
Table 2 Observed spectra of NA in the supersonic jet ( $E$ monomer and dimer 1$)$ and in argon matrix $\left(E\right.$ monomer) in the $3600-3000 \mathrm{~cm}^{-1}$ and 2000-1000 $\mathrm{cm}^{-1}$ spectral regions. B3LYP/6-311++G(d,p) theoretical vibrational data in the same spectral region is given for comparison ${ }^{a}$

\begin{tabular}{|c|c|c|c|c|c|c|c|}
\hline \multirow[b]{2}{*}{$\begin{array}{l}\text { Approximate } \\
\text { description }^{b}\end{array}$} & \multicolumn{2}{|l|}{ Dimer 1} & \multicolumn{2}{|l|}{ Monomer } & \multicolumn{2}{|l|}{ Jet } & \multirow{2}{*}{$\begin{array}{l}\text { Argon matrix } \\
\text { Monomer }\end{array}$} \\
\hline & $\begin{array}{l}\text { Calculated } \\
\text { wavenumber }\end{array}$ & $\begin{array}{l}\text { Calculated } \\
\text { intensity }\end{array}$ & $\begin{array}{l}\text { Calculated } \\
\text { wavenumber }\end{array}$ & $\begin{array}{l}\text { Calculated } \\
\text { intensity }\end{array}$ & Dimer & Monomer & \\
\hline$\nu\left(\mathrm{NH}_{2}\right)$ as & & & 3568 & 43 & & 3566 & 3555 \\
\hline$\nu(\mathrm{N}-\mathrm{H})_{\text {free }}$ & 3535 & 178 & & & 3530 & & \\
\hline$\nu\left(\mathrm{NH}_{2}\right) \mathrm{s}$ & & & 3446 & 48 & & 3449 & 3437 \\
\hline $\begin{array}{l}\nu(\mathrm{N}-\mathrm{H})_{\mathrm{H}-\text { bond }} \\
\nu(\mathrm{C}=\mathrm{O})\end{array}$ & 3204 & 2566 & 1726 & 369 & 3147 & 1726 & $1715 / 1711$ \\
\hline$\nu(\mathrm{C}=\mathrm{O})$ & 1706 & 788 & 1720 & (30 & 1707 & 1720 & $1710 / 1+11$ \\
\hline$\delta\left(\mathrm{NH}_{2}\right)$ & 1630 & 135 & & & n.o. & & \\
\hline$\delta\left(\mathrm{NH}_{2}\right)$ & & & 1601 & 142 & & 1598 & 1595 \\
\hline$\nu$ ring & 1609 & 104 & & & n.o. & & \\
\hline $\begin{array}{l}\nu \text { ring } 1 \\
v \text { ring } 5\end{array}$ & & & $\begin{array}{l}1609 \\
1587\end{array}$ & $\begin{array}{r}39 \\
6\end{array}$ & & $\begin{array}{l}\sim 1589 \\
\text { n.o. }\end{array}$ & $\begin{array}{l}1588 / 1583 \\
1573\end{array}$ \\
\hline$v$ ring & 1585 & 18 & & & n.o. & & \\
\hline$\delta(\mathrm{C}-\mathrm{H})$ & 1492 & 4 & & & n.o. & & \\
\hline$\delta(\mathrm{C}-\mathrm{H})^{\prime} 2$ & & & 1490 & 2 & & n.o. & 1483 \\
\hline $\begin{array}{l}\delta(\mathrm{C}-\mathrm{H}) \\
\delta(\mathrm{C}-\mathrm{H})^{\prime} 1\end{array}$ & 1431 & 69 & 1431 & 29 & n.o. & n.o. & $1424 / 1421$ \\
\hline $\begin{array}{l}\nu(\mathrm{C}-\mathrm{N}) \\
\nu(\mathrm{C}-\mathrm{N}) \\
\delta(\mathrm{C}-\mathrm{H})^{\prime \prime} 1\end{array}$ & 1393 & 566 & $\begin{array}{l}1348 \\
1343\end{array}$ & $\begin{array}{r}216 \\
11\end{array}$ & 1394 & 1358 & $\begin{array}{l}1370 / 1367 / 1358 / \\
1347 / 1336\end{array}$ \\
\hline
\end{tabular}

assisted by calculations carried out at DFT and MP2 levels of theory. For monomeric nicotinamide, the experimental and theoretical studies led to the identification of two conformers of the compound: the most stable $E$ form (populated by $c a$. $80 \%$ molecules of the compound) and the less stable (by $3.6 \mathrm{~kJ} \mathrm{~mol}^{-1}$ ) form $Z$ (populated by the remaining $20 \%$ of the monomers). The infrared spectra of these conformers were interpreted by comparison with their theoretically predicted counterparts.

Narrow bands due to the centrosymmetric dimer of nicotinamide were observed in the IR spectrum of the compound seeded in the supersonic jet expansion. These bands were

Table 3 Observed spectra of PA in the supersonic jet ( $E$ monomer and dimer 1$)$ and in argon matrix $\left(E\right.$ monomer) in the $3600-3000 \mathrm{~cm}^{-1}$ and $2000-1000 \mathrm{~cm}^{-1}$ spectral regions. B3LYP/6-311++G(d,p) theoretical vibrational data in the same spectral region is given for comparison ${ }^{a}$

\begin{tabular}{|c|c|c|c|c|c|c|c|}
\hline \multirow[b]{2}{*}{$\begin{array}{l}\text { Approximate } \\
\text { description }^{b}\end{array}$} & \multicolumn{2}{|l|}{ Dimer 1} & \multicolumn{2}{|l|}{ Monomer $^{19}$} & \multicolumn{2}{|l|}{ Jet } & \multirow{2}{*}{$\begin{array}{l}\text { Argon matrix } \\
\text { Monomer }^{19}\end{array}$} \\
\hline & $\begin{array}{l}\text { Calculated } \\
\text { wavenumber }\end{array}$ & $\begin{array}{l}\text { Calculated } \\
\text { intensity }\end{array}$ & $\begin{array}{l}\text { Calculated } \\
\text { wavenumber }\end{array}$ & $\begin{array}{l}\text { Calculated } \\
\text { intensity }\end{array}$ & Dimer & Monomer & \\
\hline$\nu\left(\mathrm{NH}_{2}\right)$ as & & & 3572 & 91 & & 3560 & $3546 / 3542$ \\
\hline$\nu(\mathrm{N}-\mathrm{H})_{\text {free }}$ & 3491 & 442 & & & 3475 & & \\
\hline$\nu\left(\mathrm{NH}_{2}\right) \mathrm{s}$ & & & 3436 & 46 & & 3429 & $3415 / 3414$ \\
\hline$\nu(\mathrm{N}-\mathrm{H})_{\mathrm{H}-\text { bond }}$ & 3224 & 2265 & & & 3176 & & \\
\hline$\nu(\mathrm{C}=\mathrm{O})$ & & & 1732 & 355 & & 1732 & $\begin{array}{l}1724 / 1722 / 1720 / \\
1719\end{array}$ \\
\hline$\nu(\mathrm{C}=\mathrm{O})$ & 1719 & 704 & & & 1719 & & \\
\hline$v$ ring & 1609 & 24 & & & n.o. & & \\
\hline$v \operatorname{ring} 3$ & & & 1609 & 13 & & n.o. & $1595 / 1590$ \\
\hline$\delta\left(\mathrm{NH}_{2}\right)$ & 1603 & 213 & & & n.o. & & \\
\hline$\nu$ ring 1 & & & 1592 & 24 & & n.o. & $1574 / 1572$ \\
\hline$\nu$ ring & 1591 & 79 & & & n.o. & & \\
\hline$\delta\left(\mathrm{NH}_{2}\right)$ & & & 1569 & 220 & & 1557 & $1553 / 1551$ \\
\hline $\begin{array}{l}\nu \text { ring } \\
\delta(\mathrm{C}-\mathrm{H})^{\prime} 2\end{array}$ & 1482 & 13 & 1480 & 8 & n.o. & $n \cdot 0$ & $1473 / 1469 / 1462$ \\
\hline$\delta(\mathrm{C}-\mathrm{H})$ & 1451 & 21 & & & n.o. & 11.0. & $14 / 0 / 140 / 1402$ \\
\hline$\delta(\mathrm{C}-\mathrm{H})^{\prime \prime} 1$ & & & 1449 & 6 & & n.o. & $1444 / 1442$ \\
\hline$\nu(\mathrm{C}-\mathrm{N})$ & 1398 & 451 & & & $\approx 1400$ & & \\
\hline$\nu(\mathbf{C}-\mathbf{N})$ & & & 1359 & 146 & & 1367 & $1366 / 1365$ \\
\hline
\end{tabular}

${ }^{a}$ Wavenumbers in $\mathrm{cm}^{-1}$, calculated intensities in $\mathrm{km} \mathrm{mol}^{-1} . \nu$, bond stretching, $\delta$, bending, s, symmetric, as, antisymmetric, n.o. not observed. ${ }^{b}$ See Tables S9 and S10 for full description of the normal coordinates for the dimer; for full description of the normal coordinates of the monomer, see ref. 19. 
<smiles>COC(=O)c1ccccn1</smiles>

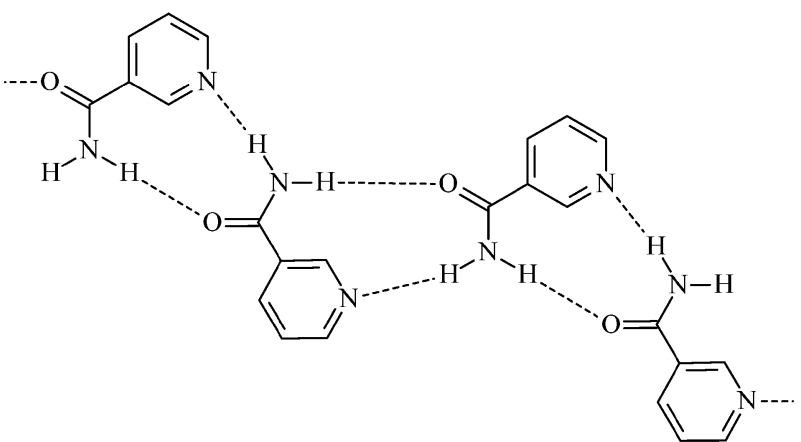

Scheme 3 Schematic representation of the H-bonds in the $\alpha$-phase crystal of picolinamide (top) and nicotinamide (bottom).

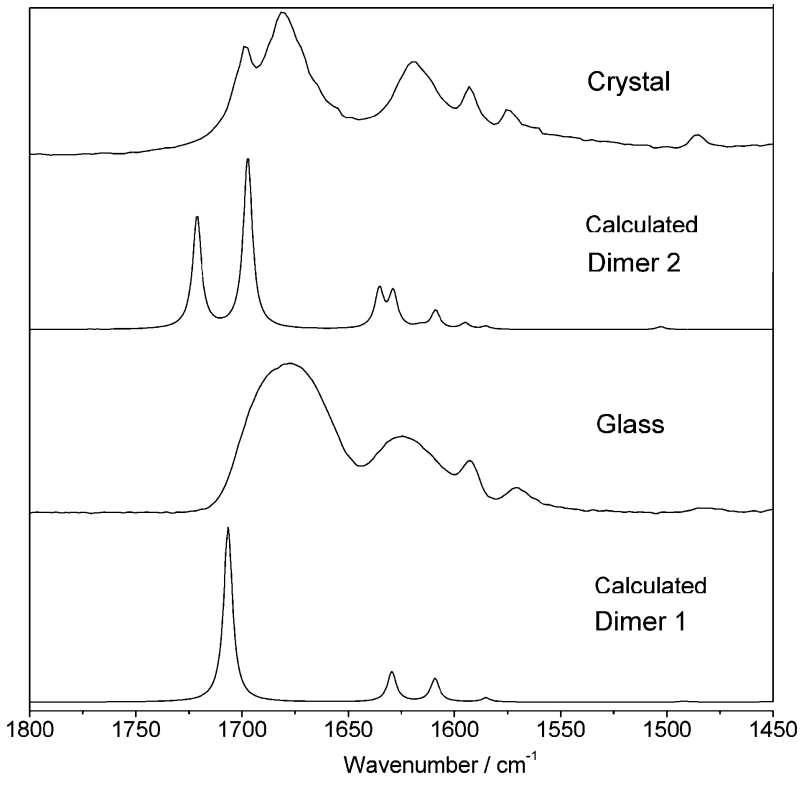

Fig. $71800-1450 \mathrm{~cm}^{-1}$ region of the infrared spectra of nicotinamide. From the bottom to the top: B3LYP/6-311+ + G(d,p) calculated IR spectrum for the centrosymmetric dimer (dimer 1) (scaled by 0.988), spectrum of the glassy state obtained by fast deposition of the vapour of the compound onto the cold tip of the cryostat $(10 \mathrm{~K})$, calculated spectrum for dimer 2 and spectrum of the room temperature crystalline state $\left(\mathrm{KBr}\right.$ pellet; $\left.25^{\circ} \mathrm{C}\right)$.

characteristically shifted with respect to the IR absorptions of nicotinamide monomers. For picolinamide, the most

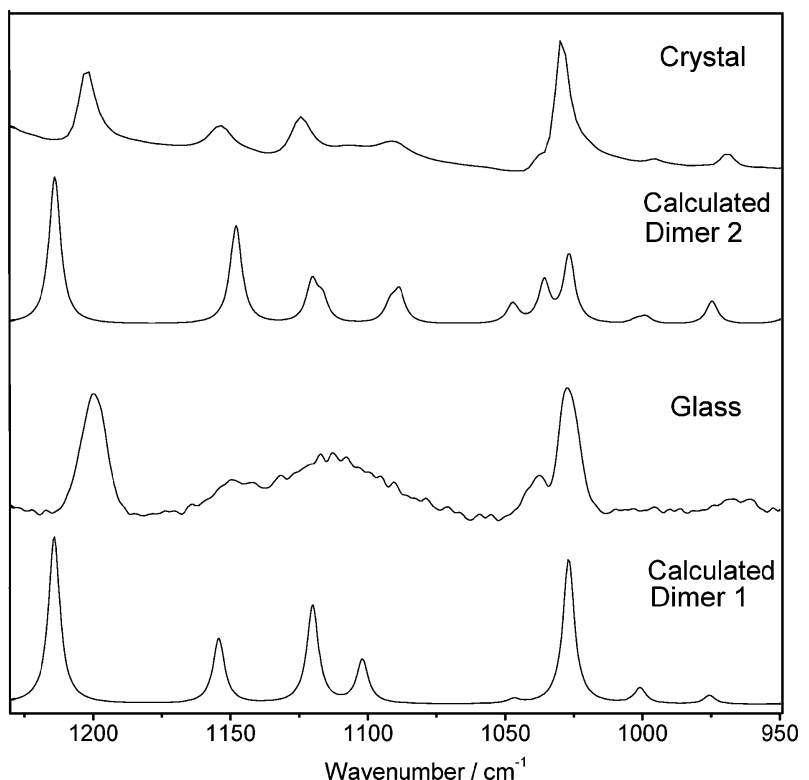

Fig. $8 \quad 1230-950 \mathrm{~cm}^{-1}$ region of the infrared spectra of nicotinamide. From the bottom to the top: B $3 \mathrm{LYP} / 6-311++\mathrm{G}(\mathrm{d}, \mathrm{p})$ calculated IR spectrum for the centrosymmetric dimer (dimer 1) (scaled by 0.988 ), spectrum of the glassy state obtained by fast deposition of the vapour of the compound onto the cold tip of the cryostat $(10 \mathrm{~K})$, calculated spectrum for dimer 2 and spectrum of the room temperature crystalline state $\left(\mathrm{KBr}\right.$ pellet; $\left.25^{\circ} \mathrm{C}\right)$.

red-shifted $\mathrm{N}-\mathrm{H}$ dimer band is significantly broadened, which is ascribed to accelerated IVR via the second $\mathrm{N}-\mathrm{H}$ bond and a weak intramolecular hydrogen bond contact into the heterocyclic frame.

The structures of aggregates of nicotinamide and picolinamide, constituting the building blocks of condensed phases of the compounds, were accounted for on the basis of the infrared spectra recorded for low-temperature glass and crystalline states of these species. These studies led to identification of the most stable centrosymmetric dimers as the most populated structural motifs of both nicotinamide and picolinamide in the glassy state. These structural units (centrosymmetric dimers) were also found to build the crystalline state of picolinamide. However, it was demonstrated that in crystalline nicotinamide, dimers other than centrosymmetric are formed. This was revealed by the small changes noticed in the infrared spectrum of picolinamide upon transition from glassy to crystalline state and by the much more pronounced spectral changes accompanying the analogous phase transition in solid nicotinamide. These spectral investigations are in accordance with the previous crystallographic studies on nicotinamide and picolinamide.

\section{Acknowledgements}

This work was supported by FCT (Projects POCTI/QUI/ 59019/2004 and POCTI/QUI/58937/2004) and a DFG research training group (http://www.pcgg.de). The authors also thank DAAD and FCT/GRICES for supporting the bilateral collaboration between the Portuguese and German 
groups. A.B. acknowledges FCT the $\mathrm{PhD}$ grant (SFRH/BD/ $21543 / 2005)$.

\section{References}

1 R. B. Elliott, C. C. Pilcher and A. Stewart, Ann. N. Y. Acad. Sci., 1993, 696, 333.

2 M. T. Behme, Nutr. Rev., 1995, 53, 137.

3 J. P. Kamat and T. P. Devasagayam, Redox Rep., 1999, 4, 179.

4 R. W. Pero, B. Axelsson and D. Siemann, Mol. Cell. Biochem., 1999, 193, 119.

5 A. R. Shalita, J. G. Smith and L. C. Parish, Int. J. Dermatol., 1995, 34, 434.

6 C. Griffiths, J. Dermatol. Treat., 1995, 6(S8), 10.

7 L. K. Klaidman, S. K. Mukherjee, T. P. Hutchin and J. D. Adams, Neurosci. Lett., 1996, 206, 5.

8 E. L. Jacobson, W. M. Shieh and A. C. Huang, Mol. Cell. Biochem., 1999, 193, 69.

9 J. M. Denu, Biochem. Sci., 2005, 30, 479.

$10 \mathrm{H}$. Yamamoto and H. Okamoto, Biochem. Biophys. Res. Commun., 1980, 95, 474.

11 T. Takeshima, H. Takeuchi, T. Egawa and S. Konaka, J. Mol. Struct., 2003, 644, 197.

12 B. Vogelsanger, R. D. Brown, P. D. Godfrey and A. P. Pierlot, J. Mol. Spectrosc., 1991, 145, 1.

13 H. Shindo, Pharm. Bull. (Tokyo), 1957, 5, 472.

14 E. A. Velcheva and L. I. Daskalova, J. Mol. Struct., 2005, 741, 85.

15 L. I. Daskalova, E. A. Velcheva and I. G. Binev, J. Mol. Struct., 2007, 826, 198.

16 M. Bakiler, O. Bolukbasi and A. Yilmaz, J. Mol. Struct., 2007, 826, 6.

17 P. Purcell and J. A. Singer, J. Phys. Chem., 1965, 69, 4097.

18 J. Kuthan, L. Musil and V. Jehlicka, Collect. Czech. Chem. Commun., 1977, 42, 283.

19 A. Borba, A. Gómez-Zavaglia and R. Fausto, J. Phys. Chem. A, 2008, 112, 45.

20 N. C. Singa and D. N. Sathyanarayana, Spectrochim. Acta A, 1998, 54, 1059.

21 J. Anad, N. C. Singha and D. N. Sathyanarayana, J. Mol. Struct., 1997, 412, 221.

22 B. Wright and G. S. D. King, Acta Crystallogr., 1954, 7, 283.

23 Y. Miwa, T. Mizuno, K. Tsuchiya, T. Taga and Y. Iwata, Acta Crystallogr., Sect. B, 1999, 55, 78.

24 T. Takano, Y. Sasada and M. Kakudo, Acta Crystallogr., 1966, 21, 514.
25 E. G. Robertson, M. R. Hockridge, P. D. Jelfs and J. P. Simons, Phys. Chem. Chem. Phys., 2001, 3, 786.

26 Y. Matsuda, T. Ebata and N. Mikami, J. Chem. Phys., 1999, 110, 8397.

27 M. Albrecht, C. A. Rice and M. A. Suhm, J. Phys. Chem. A, 2008, 112, 7530 .

28 D. W. Vidrine and C. A. Anderson, Silicon Beamsplitter, U.S. Patent 4,632,553, 1986.

29 T. Häber, U. Schmitt and M. A. Suhm, Phys. Chem. Chem. Phys., 1999, 1, 5573.

30 C. Cézard, C. A. Rice and M. A. Suhm, J. Phys. Chem. A, 2006, $110,9839$.

31 M. J. Frisch, G. W. Trucks, H. B. Schlegel, G. E. Scuseria, M. A. Robb, J. R. Cheeseman, J. A. Montgomery, T. Vreven, K. N. Kudin, J. C. Burant, J. M. Millam, S. S. Iyengar, J. Tomasi, V. Barone, B. Mennucci, M. Cossi, G. Scalmani, N. Rega, G. A. Petersson, H. Nakatsuji, M. Hada, M. Ehara, K. Toyota, R. Fukuda, J. Hasegawa, M. Ishida, T. Nakajima, Y. Honda, O. Kitao, H. Nakai, M. Klene, X. Li, J. E. Knox, H. P. Hratchian, J. B. Cross, V. Bakken, C. Adamo, J. Jaramillo, R. Gomperts, R. E. Stratmann, O. Yazyev, A. J. Austin, R. Cammi, C. Pomelli, J. W. Ochterski, P. Y. Ayala, K. Morokuma, G. A. Voth, P. Salvador, J. J. Dannenberg, V. G. Zakrzewski, S. Dapprich, A. D. Daniels, M. C. Strain, O. Farkas, D. K. Malick, A. D. Rabuck, K. Raghavachari, J. B. Foresman, J. V. Ortiz, Q. Cui, A. G. Baboul, S. Clifford, J. Cioslowski, B. B. Stefanov, G. Liu, A. Liashenko, P. Piskorz, I. Komaromi, R. L Martin, D. J. Fox, T. Keith, M. A. Al-Laham, C. Y. Peng, A. Nanayakkara, M. Challacombe, P. M. W. Gill, B. Johnson, W. Chen, M. W. Wong, C. Gonzalez and J. A. Pople, GAUSSIAN 03 (Revision C.02), Gaussian Inc., Wallingford, CT, 2004.

32 A. Becke, Phys. Rev. A, 1988, 38, 3098.

33 C. Lee, W. Yang and R. Parr, Phys. Rev. B., 1988, 37, 785.

34 S. Vosko, L. Wilk and M. Nusair, Can. J. Phys., 1980, 58, 1200.

35 C. Møller and M. S. Plesset, Phys. Rev., 1934, 46, 618.

36 M. Frisch, M. Head-Gordon and J. Pople, Chem. Phys. Lett., 1990, 166, 281.

37 J. Schachtschneider, Technical Report; Shell Development Co. Emeryville, CA, 1969.

38 P. Carbonniere, T. Lucca, C. Pouchan, N. Rega and V. Barone, J. Comput. Chem., 2005, 26, 384.

39 M. Szafran and J. Koput, J. Mol. Struct., 2001, 555/556, 439.

40 A. J. Barnes, J. Mol. Struct., 1984, 113, 161.

41 M. Albrecht, P. Zielke, C. A. Rice and M. A. Suhm, J. Mol. Struct., 2008, 880, 2.

42 T. N. Wassermann, C. A. Rice, M. A. Suhm and D. Luckhaus, J. Chem. Phys., 2007, 127, 234309. 